\title{
Atom Probe Characterization of Corroded Alloy 600
}

\author{
Baptiste Gault ${ }^{1,2}$, Fabio Scenini ${ }^{3}$, Michael P. Moody ${ }^{4}$, Julia H. Huang ${ }^{5}$, Gianluigi A. Botton ${ }^{1,5}$, \\ Dominique Mangelinck $^{6}$, Marion Descoins ${ }^{6}$, Roger C. Newman ${ }^{7}$ \\ ${ }^{1}$ Department of Materials Science \& Engineering, McMaster University, Hamilton, ON, Canada \\ 2. now at Elsevier Ltd, Kidlington, UK \\ ${ }^{3}$ Materials Performance Centre, University of Manchester, Manchester, uk \\ ${ }^{4}$ Department of Materials, University of Oxford, Oxford, UK \\ ${ }^{5}$ Canadian Centre for Electron Microscopy, Brockhouse Institute, McMaster University, Hamilton, ON, \\ Canada \\ ${ }^{6}$ IM2NP, UMR CNRS 7334 - Université Aix-Marseille, Marseille, France \\ ${ }^{7}$ Department of Chemical Engineering \& Applied Chemistry, University of Toronto, Toronto, ON, \\ Canada
}

Internal oxidation is believed to be the main cause for primary water stress corrosion cracking in nickelbased Alloy 600 [1]. In order to elucidate the corrosion mechanism, we used atom probe tomography (APT) to investigate the near-surface nanostructure, within grains and at grain boundaries, of an Alloy 600 sample corroded in hydrogenated steam at $480^{\circ} \mathrm{C}$ to mimic the behavior in service (hydrogenated water at ca. $320-330^{\circ} \mathrm{C}$ ). The composition of the alloy is reported in Table 1 . The samples were prepared from a mill-annealed tube that was solution-annealed at a higher than usual temperature to grow the grain size, and were oxidized for $96 \mathrm{~h}$ at a calculated (effective) partial pressure of $\mathrm{O}_{2}$ very close to the dissociation pressure of $\mathrm{NiO}$.

Specimens for APT from the near-surface of the sample were prepared with a FIB/SEM using the conventional lift-out procedure [2]. To investigate the internal oxidation we targeted a region (ROI-1) in the core of a severely oxidized grain showing a high surface roughness, as shown in Figure 1 (a). We then targeted the region between two grains exhibiting different surface structures (ROI-2), as depicted in Figure 1(b), to unveil the chemistry of the boundary. APT experiments were performed on two different Cameca LEAP $3000 \mathrm{X}$ HR, in laser-pulsing mode $(200 \mathrm{kHz}, 532 \mathrm{~nm}, 0.6-0.8 \mathrm{~nJ})$, at a base temperature of $60 \mathrm{~K}$, and an average detection flux of $5 \times 10^{-3} \mathrm{ion} /$ pulse. Datasets containing 5-30 $\mathrm{M}$ ions were acquired and reconstructed using the IVAS software suite. When possible the reconstruction was calibrated based on the pole figure and interplanar spacing [3].

The data from ROI-1, as shown in Figure 1(b), shows large Cr-rich oxide-particles. A population of smaller oxide particles are also visible in the data, as highlighted by the purple isoconcentration surface in Figure 1(b). APT allows for a precise characterization of the solute distribution between the oxide and the alloy, and we demonstrate that B and V segregate to the interface between the oxide and the alloy, while the Fe seem to almost equally partition between the oxide and the alloy. In the data from ROI-2, the significant enrichment in $\mathrm{Ni}$ is evidenced in a region surrounding the grain boundary. In this dataset, atomic planes are imaged, as shown in Figure 2(b), and the Ni-concentration reaches almost 80 at $\%$, compared to about 60 at\% within the alloy. We demonstrate here that $\mathrm{Nb}$ is segregated to the grain boundary, as shown in Figure 2(c). From complementary electron microscopy studies, the clusters observed on the surface in Figure 1 (a) and (b) appear to be almost pure Ni. It is postulated that these are formed by short-circuit diffusion of $\mathrm{Ni}$ to the surface, consequent on the compressive stress induced by internal oxidation of $\mathrm{Cr}$. 


\section{References:}

[1] F. Scenini, R.C. Newman et al. , Corrosion, 64 (2008) 824-835.

[2] K. Thompson et al. Ultramicroscopy, 107 (2007) 131-139.

[3] B. Gault, M.P. Moody et al. Journal of Applied Physics, 105 (2009) 034913.

[4] BG acknowledges the Canadian Centre for Electron Microscopy for access to the FIB, and METSA platform for supporting access to the FIB and the LEAP in Marseille.

\begin{tabular}{ccccccccc}
\hline $\mathbf{C}$ & $\mathbf{N i}$ & $\mathbf{C r}$ & $\mathbf{F e}$ & $\mathbf{M n}$ & $\mathbf{S i}$ & $\mathbf{C u}$ & $\mathbf{S}$ & $\mathbf{C o}$ \\
$\mathbf{0 . 0 3 8}$ & Bal. & 15.86 & 8.07 & 0.26 & 0.33 & 0.01 & 0.001 & 0.021 \\
\hline
\end{tabular}

Table 1: composition of the alloy (wt \%).

(b)

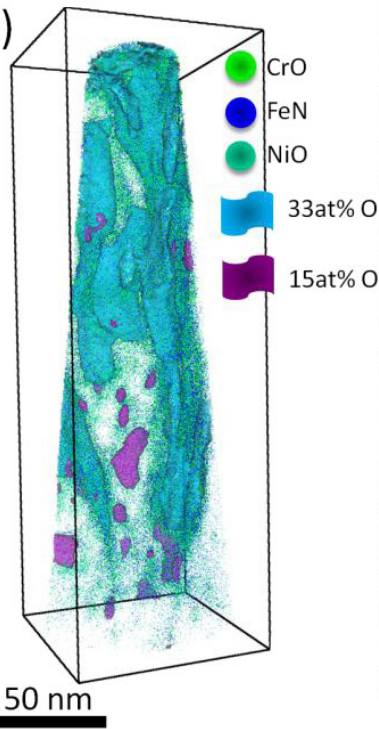

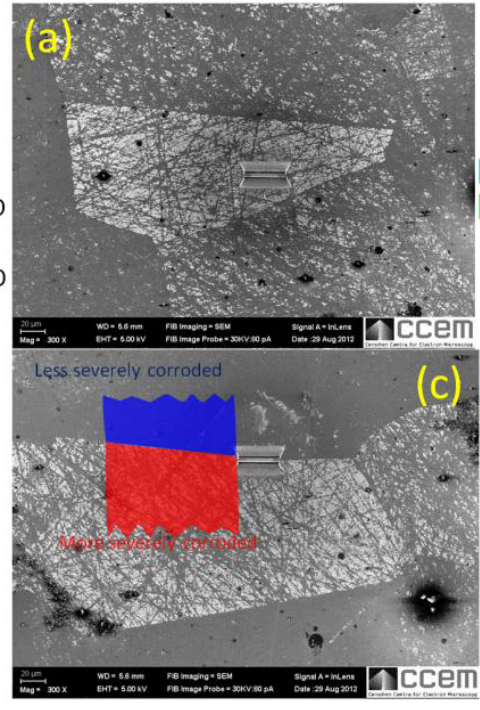

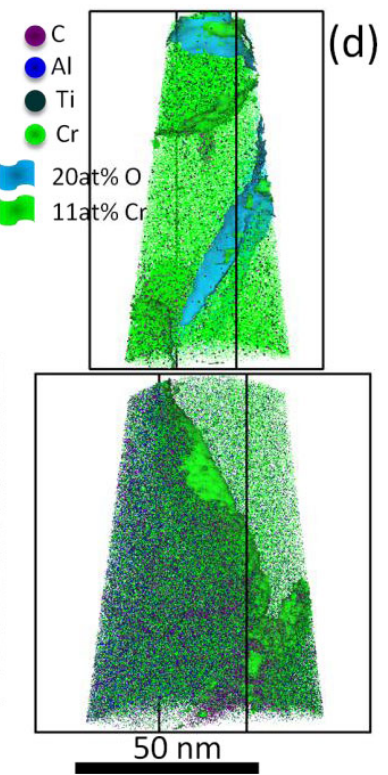

Figure 1 (a) SEM image of the corroded surface. The lift-out bar (ROI-1) is visible in the centre of the grain. (b) Typical APT dataset obtained from the centre of the grain. A network of interconnected oxide particles can be seen along with smaller oxide particles farther below the surface. (c-d) Picked boundary and APT datasets (ROI-2), the grain boundary is highlighted by the blue isosurface, and it is within the high-Ni (low-Cr) region contained within the top and bottom green isoconcentration surfaces.
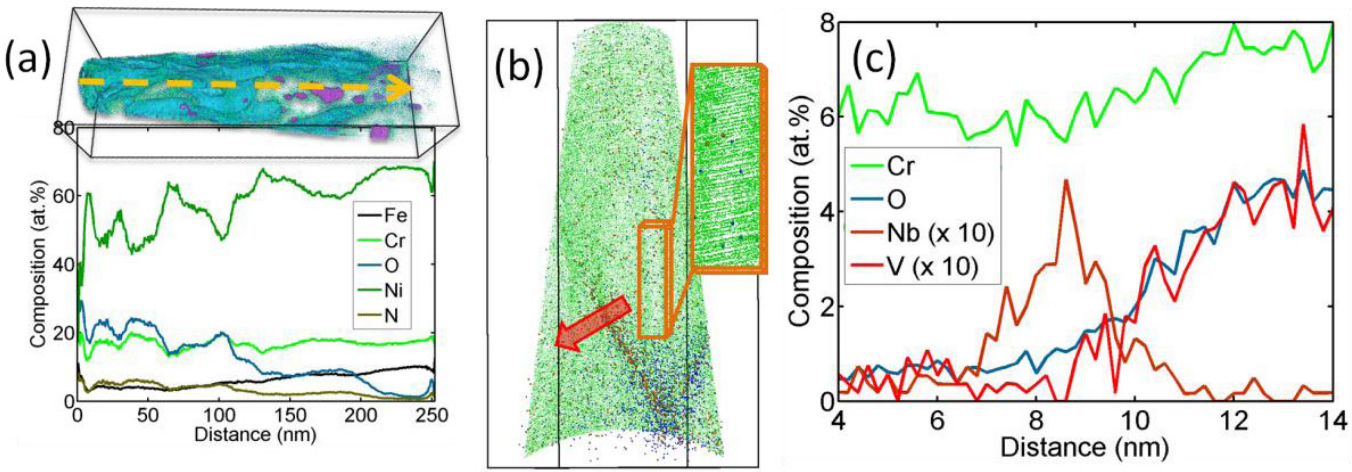

Figure 2. (a) Composition profile along the dataset shown in Figure 1(a) showing the progressive decay of the Oxygen concentration. (b) Top dataset from Figure 1(b) containing the grain boundary. Inset is a close-up on the atomic planes imaged down to the grain boundary. (c) Composition profile through the boundary highlights the segregation of $\mathrm{Nb}$, as well as the slight change in composition between the grains. 